ARTICLE

DOI: $10.1038 / s 41467-017-00107-4$

OPEN

\title{
Impact of interfacial molecular orientation on radiative recombination and charge generation efficiency
}

Niva A. Ran¹, Steffen Roland ${ }^{2}$, John A. Love ${ }^{1,2,3}$, Victoria Savikhin ${ }^{4,5}$, Christopher J. Takacs 3,4 , Yao-Tsung Fu ${ }^{6}$, Hong Li ${ }^{6}{ }^{6}$, Veaceslav Coropceanu ${ }^{6}$, Xiaofeng Liu', Jean-Luc Brédas ${ }^{6}$, Guillermo C. Bazan', Michael F. Toney (10 ${ }^{4}$, Dieter Neher ${ }^{2} \&$ Thuc-Quyen Nguyen ${ }^{1}$

A long standing question in organic electronics concerns the effects of molecular orientation at donor/acceptor heterojunctions. Given a well-controlled donor/acceptor bilayer system, we uncover the genuine effects of molecular orientation on charge generation and recombination. These effects are studied through the point of view of photovoltaics-however, the results have important implications on the operation of all optoelectronic devices with donor/ acceptor interfaces, such as light emitting diodes and photodetectors. Our findings can be summarized by two points. First, devices with donor molecules face-on to the acceptor interface have a higher charge transfer state energy and less non-radiative recombination, resulting in larger open-circuit voltages and higher radiative efficiencies. Second, devices with donor molecules edge-on to the acceptor interface are more efficient at charge generation, attributed to smaller electronic coupling between the charge transfer states and the ground state, and lower activation energy for charge generation.

\footnotetext{
${ }^{1}$ Center for Polymers and Organic Solids, University of California, Santa Barbara, CA 93106, USA. ${ }^{2}$ Institute of Physics and Astronomy, University of Potsdam, Potsdam 14476, Germany. ${ }^{3}$ Materials Department, University of California, Santa Barbara, CA 93106, USA. ${ }^{4}$ Stanford Synchrotron Radiation Lightsource, SLAC National Accelerator Laboratory, Menlo Park, CA 94025, USA. ${ }^{5}$ Department of Electrical Engineering, Stanford University, Stanford, CA 94305, USA. ${ }^{6}$ School of Chemistry and Biochemistry and Center for Organic Photonics and Electronics, Georgia Institute of Technology, Atlanta, GA 30332, USA. Correspondence and requests for materials should be addressed to T.-Q.N. (email: quyen@chem.ucsb.edu)
} 
T he efficiencies of charge generation and recombination at a donor acceptor heterojunction depend on parameters, such as distance and molecular orientation of the donor and acceptor molecules at the interface. These processes dictate the performance of electronic devices such as light emitting diodes (LEDs), photodetectors, and photovoltaics. It is therefore critical to understand the properties of the donor/acceptor interface which affect the efficiencies of charge generation and recombination. The properties of the donor/acceptor interface can be studied from the point of view of photovoltaics, with implications on the performance of other devices which depend on donor/ acceptor interfaces.

A fundamental issue under much debate in the organic photovoltaic (OPV) literature involves the geometry of the donor/ acceptor interface: whether a face-on geometry (one where the $\pi$-faces of the donor and acceptor $\pi$-conjugated molecules or polymer chains are parallel) is favorable compared to an edge-on geometry (where the $\pi$-faces are orthogonal). Theoretical calculations have long suggested that the nature of the donor-acceptor interface will have a large effect on the rates of charge transfer and recombination $^{1-3}$, as well as charge delocalization ${ }^{3}, 4$. Other calculations have found that molecular orientation affects interfacial quadrupoles and consequently the ease of charge separation ${ }^{5,6}$. A number of researchers have attempted to resolve this question experimentally with the use of controlled donor and/or acceptor orientations in planar heterojunction solar cells ${ }^{1,7-14}$. In general, most studies have found that within the same material system, face-on solar cells have a superior power conversion efficiency (PCE) when compared to the edge-on orientation ${ }^{7,15}$. This has been attributed primarily to changes in the donor ionization potential (IP) (or, to a first approximation, highest occupied molecular orbital energy level), which directly affects the open circuit voltage $\left(V_{\mathrm{OC}}\right)^{7-9,11,15-17}$, but has also been explained by differences in recombination rates ${ }^{1,7,12,15}$.

Despite significant efforts, it has remained very challenging to fabricate high-quality planar heterojunctions with identical active layers and contacts, but opposite molecular orientations. Most studies settle for comparisons between one orientation and a mixed orientation, or modified contacts to induce changes in orientation. Furthermore, it was demonstrated that molecular diffusion in planar heterojunctions can happen spontaneously ${ }^{18}$, and to varying extents for different orientations ${ }^{19}$. Interfacial mixing will lead to unfair comparisons and erroneous conclusions if not properly taken into account. Furthermore, differences due to interfacial and/or bulk disorder have also been shown to influence device performance ${ }^{20,21}$. To date, experimental studies in which the effects of molecular orientation have truly been isolated-a single materials system in which the two extremes of face-on and edge-on orientations can be accessed while maintaining abrupt donor/acceptor interfaces and identical contacts-have not been reported.

From the point of view of molecular orientation, our study addresses two topics that have been gaining significant attention in the OPV literature: non-radiative recombination losses to the $V_{\mathrm{OC}}{ }^{3,22-27}$, and the driving force for charge generation ${ }^{28-30}$. It is thought that non-radiative recombination plays a significant role in efficiency losses in photovoltaics and LEDs, and it is only when non-radiative pathways have been eliminated that organic solar cells become competitive with high performance inorganic materials $^{23}, 25,31,32$. A recent theoretical study by Chen et al. on pentacene/ $\mathrm{C}_{60}$ interfaces predicts that face-on interactions result in less non-radiative recombination, due to reduced vibronic coupling between the charge transfer (CT) state and the ground state $(\mathrm{GS})^{3}$. To date, little is understood about the origin of nonradiative recombination in organic solar cells, how it relates to molecular orientation, or how to curtail this recombination pathway. Furthermore, the driving force for charge generation has remained a disputed topic in the literature, with researchers quoting the need for energetic offsets ${ }^{33}, 34$, hot charges $^{35}, 36$, delocalization ${ }^{37,38}$, low reorganization energies ${ }^{39}$, electric fields ${ }^{40,41}$, energetic cascades and disorder ${ }^{42,} 43$, and entropy ${ }^{29,} 44$, to achieve efficient charge generation.

In this work, we begin by establishing that we are able to precisely control the molecular orientation of p-SIDT $\left(\text { FBTTh }_{2}\right)_{2}{ }^{45}$ (structure and orientations shown in Fig. 1a) in neat films, and fabricate bilayer heterojunctions with sharp, welldefined interfaces of known molecular orientations. We then analyze the photovoltaic performance, which reveals that molecular orientation has a profound effect on the $V_{\mathrm{OC}}$ and shortcircuit current $\left(J_{\mathrm{SC}}\right)$ (Fig. 1b). The higher $V_{\mathrm{OC}}$ of the solar cells with face-on p-SIDT $\left(\text { FBTTh }_{2}\right)_{2}$ is attributed to a higher CT state energy $\left(E_{\mathrm{CT}}\right)$ and lower non-radiative recombination losses. However, the edge-on solar cells are more efficient at charge generation, illustrated by a higher internal quantum efficiency (IQE). Electronic-structure calculations predict that the face-on bilayers have a larger electronic coupling between the CT state and GS, suggesting that they suffer from greater geminate recombination. In addition, charge generation in face-on bilayers is significantly more temperature-dependent than edge-on bilayers, which may be a consequence of a larger barrier to charge generation or favorable polarization at the edge-on donor/ acceptor interface. Our study presents important experimental evidence pertaining to the effect of molecular orientation on nonradiative recombination and the efficiency of charge generation.
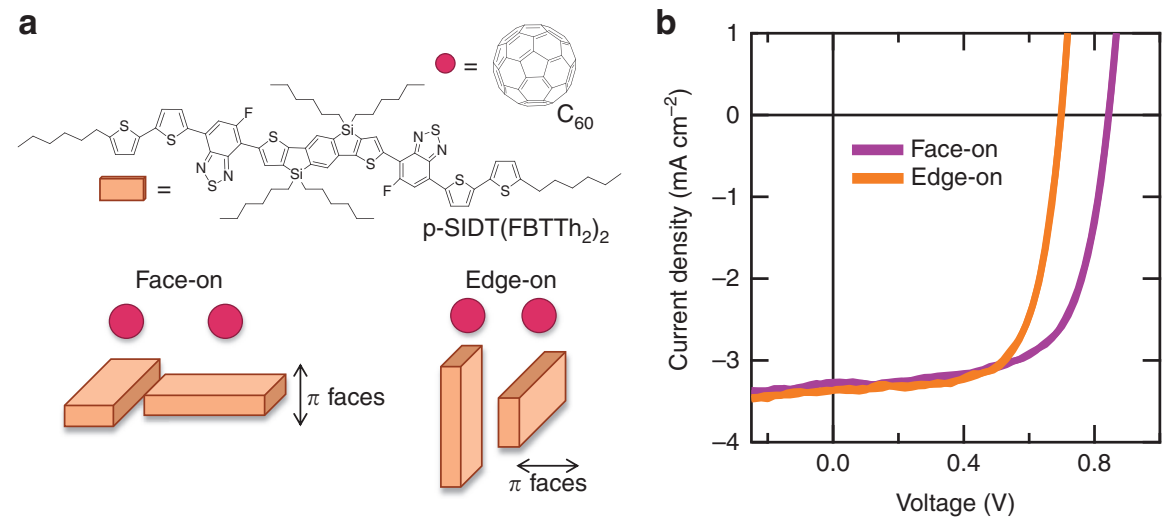

Fig. 1 Molecular orientation and the resulting photovoltaic performance. a Molecular structures of p-SIDT(FBTTh $)_{2}$ and $\mathrm{C}_{60}$ along a cartoon depicting the relative orientations of the two molecules in the face-on and edge-on bilayer samples; $\mathbf{b}$ Current-voltage characteristics of bilayer devices under 1 sun illumination 
a

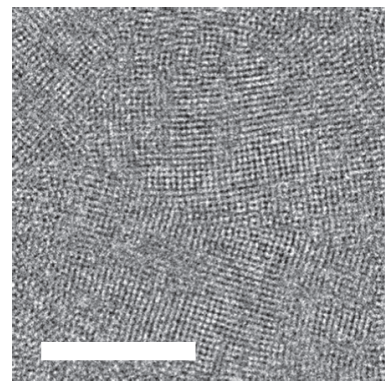

C

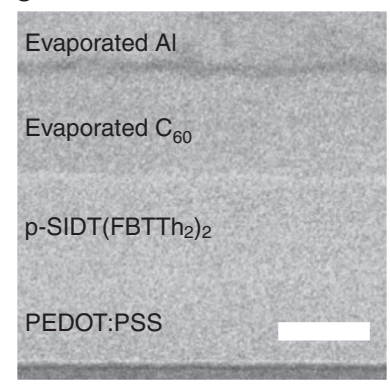

b

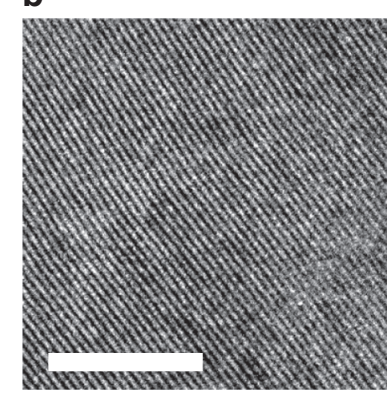

d

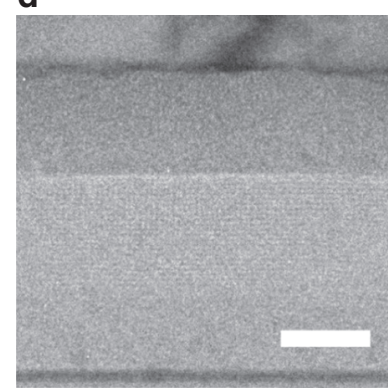

e

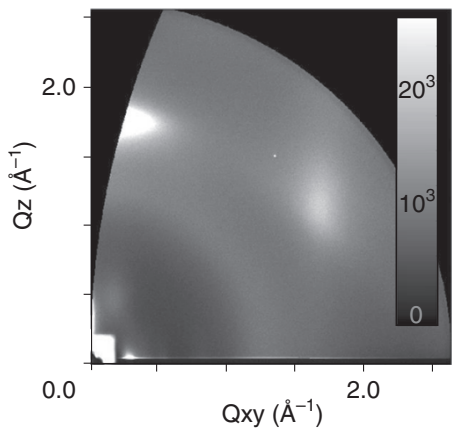

g

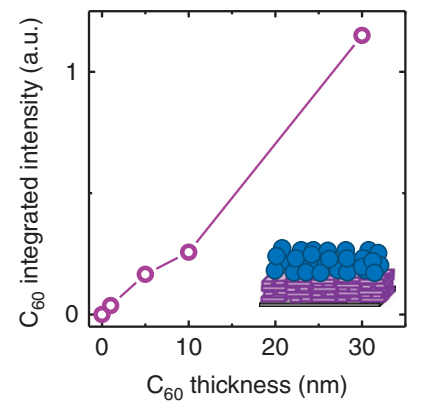

$\mathbf{f}$

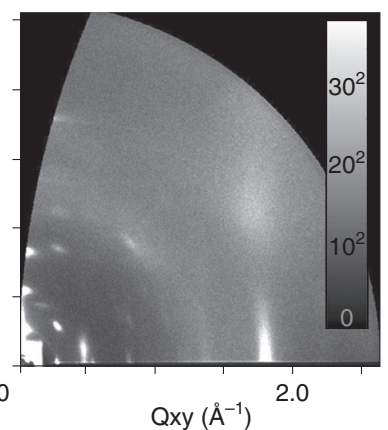

h

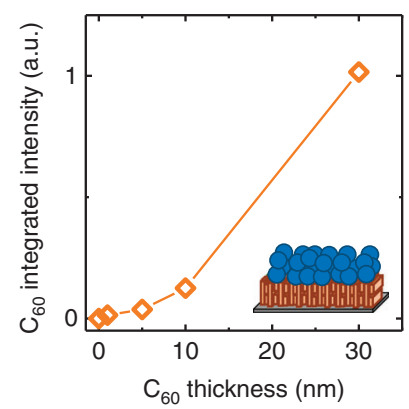

Fig. 2 Structural characterization of the molecular orientation and interface quality. HR-TEM images (a, $\mathbf{b})$ and GIWAXS spectra (e, f) of face-on (a, e) and edge-on (b, f) p-SIDT $\left(F B T T h_{2}\right)_{2}$ films. Cross-section HR-TEM of face-on (c) and edge-on (d) bilayers. Correlation of peak intensity fitting with $\mathrm{C}_{60}$ thickness evaporated on films of p-SIDT(FBTTh $)_{2}$ for face-on ( $\mathbf{g}$ ) and edge-on (h) samples. For more detail, refer to Supplementary Fig. 3. Scale bar for the HR-TEM images is $50 \mathrm{~nm}$; scale bar for the cross-section HR-TEM images is $40 \mathrm{~nm}$

\section{Results}

Structural characterization. To begin, films of neat $\mathrm{p}$-SIDT $\left(\mathrm{FBTTh}_{2}\right)_{2}$ were characterized to quantify bulk and interfacial molecular orientation. Preferential orientation can be measured using grazing incidence wide-angle X-ray scattering (GIWAXS), by comparing intensities of in-plane and out-of-plane $\pi$-stacking peaks (at $q \sim 1.7 \AA^{-1}$ ). More detail on the methodology is available in the Supplementary Fig. 1. When cast from chlorobenzene (CB), films of p-SIDT $\left(\text { FBTTh }_{2}\right)_{2}$ show a ratio of 99.5:0.5 face-on: edge-on orientation. When cast from $\mathrm{CB}$ with $0.4 \% \mathrm{v} / \mathrm{v}$ diiodooctane (DIO), films of p-SIDT(FBTTh $\left.{ }_{2}\right)_{2}$ show a ratio of 94:6 edge-on:face-on orientation. The two orientations also have distinctly different structures seen by high-resolution transmission electron microscope (HR-TEM) images. Figure 2a-d show HR-TEM and GIWAXS images of p-SIDT $\left(\mathrm{FBTTh}_{2}\right)_{2}$ films cast from $\mathrm{CB}$ (face-on) and $\mathrm{CB}+\mathrm{DIO}$ (edge-on).

In addition to providing information about the molecular orientation, the GIWAXS images in Figs. $2 c$, $d$ also show that the edge-on p-SIDT $\left(\text { FBTTh }_{2}\right)_{2}$ films are more ordered than the faceon films. We do not believe that this difference in ordering has a large impact on the results discussed further in this study; we refer the reader to further data and discussion on this topic in Supplementary Fig. 2 and Supplementary Notes 1, 2.

The quality of the $\mathrm{p}$-SIDT(FBTTh $\left.{ }_{2}\right)_{2}$ interface was verified by cross-section HR-TEM and GIWAXS. The miscibility of $\mathrm{C}_{60}$ into p-SIDT $\left(\mathrm{FBTTh}_{2}\right)_{2}$ was determined by monitoring the GIWAXS signal of varying thicknesses of $\mathrm{C}_{60}$ evaporated on p-SIDT $\left(\mathrm{FBTTh}_{2}\right)_{2}$ (Supplementary Fig. 3). A linear increase in $\mathrm{C}_{60}$ scattering intensity with $\mathrm{C}_{60}$ thickness indicates that $\mathrm{C}_{60}$ molecules are not diffusing into the p-SIDT $\left(\mathrm{BFTTh}_{2}\right)_{2}$ layer beneath. Figure $2 \mathrm{~g}$ shows a linear signal growth with $\mathrm{C}_{60}$ thickness for the face-on device, confirming that the interface is sharp. The trace for the edge-on device, Fig. $2 \mathrm{~h}$, shows a slight deviation from linearity at small thicknesses of $\mathrm{C}_{60}$. However, this can be explained by deposition of $\mathrm{C}_{60}$ into pinholes in the edgeon p-SIDT(FBTTh $)_{2}$ film which are not present in the face-on film (Supplementary Fig. 4). In addition to reducing the actual film thickness from the nominal predicted film thickness, the surface topography presented by pinholes may have an unpredictable effect on the X-ray scattering. Cross-section TEM (Figs. 2e, f) shows no evidence of interdiffusion for either face-on or edge-on bilayers. In fact, lattice planes can be well resolved in the cross-section TEM of the edge-on sample for the entire thickness of the p-SIDT $\left(\mathrm{FBTTh}_{2}\right)_{2}$ layer (Fig. 2f). The lattice planes further confirm that the donor layers retain their orientation through the bulk of the film to the interface with $\mathrm{C}_{60}$, and that deposition of the $\mathrm{C}_{60}$ layer does not disrupt the packing of $\mathrm{p}$-SIDT(FBTTh $\left.{ }_{2}\right)_{2}$. Thus, it can be concluded that the donor/acceptor interface is abrupt for edge-on and face-on p$\operatorname{SIDT}\left(\mathrm{FBTTh}_{2}\right)_{2}$.

Solar cell characteristics. The $J-V$ characteristics of the edge-on and face-on bilayers, using identical contacts (ITO/PEDOT:PSS/ active layer/BCP/Al, see Methods for full material names), under 1 sun illumination, are presented in Fig. $1 \mathrm{~b}$ and Table 1. The $J_{\mathrm{SC}}$ and the fill factor (FF) are very similar for both molecular

\begin{tabular}{|c|c|c|c|}
\hline & $v_{\text {oC }}(\mathrm{V})$ & $J_{S C}\left(m A / c^{2}\right)$ & FF (\%) \\
\hline $\begin{array}{l}\text { Face-on } \\
\text { Edge-on }\end{array}$ & $\begin{array}{l}0.84 \pm 0.03 \\
0.69 \pm 0.04\end{array}$ & $\begin{array}{l}-2.97 \pm 0.3 \\
-3.03 \pm 0.4\end{array}$ & $\begin{array}{l}66 \pm 5 \\
68 \pm 3\end{array}$ \\
\hline
\end{tabular}



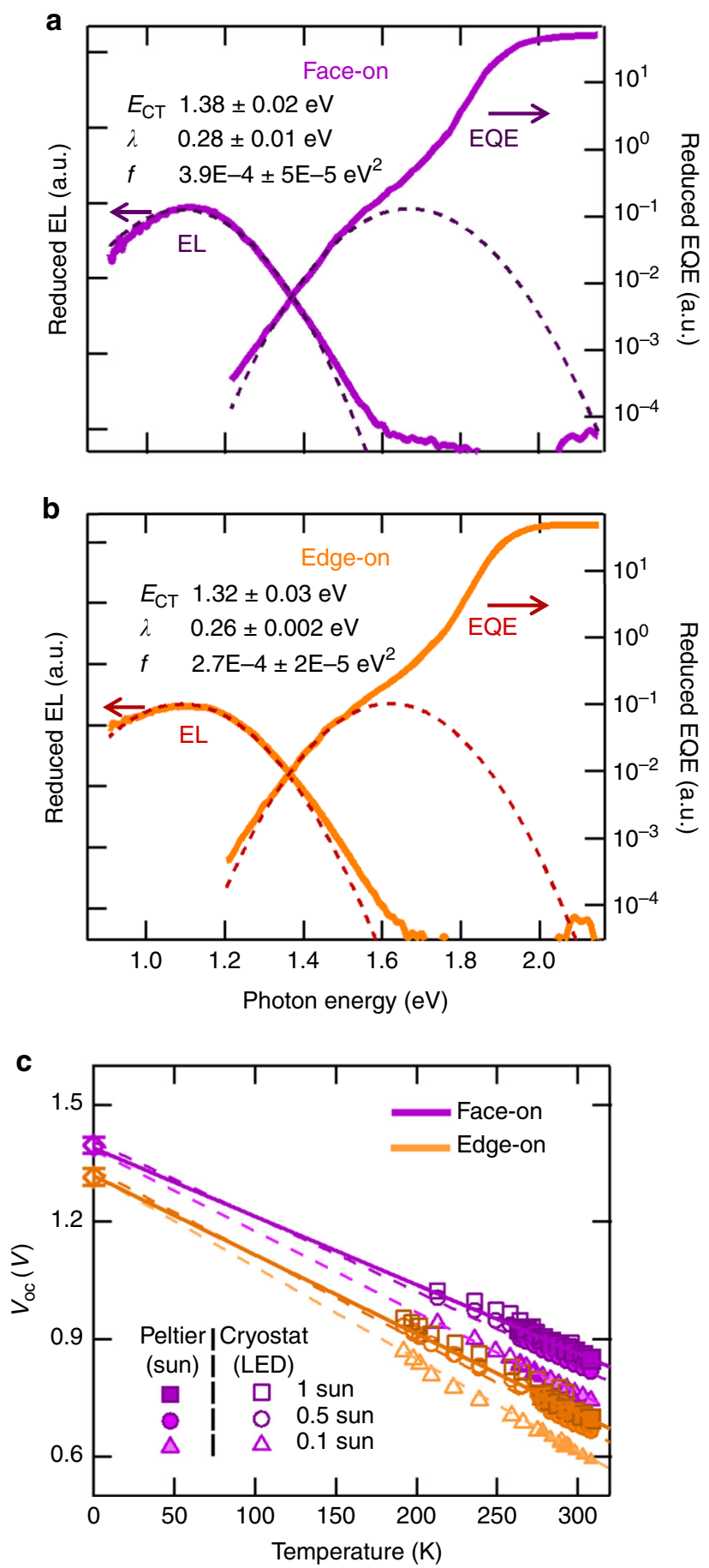

Fig. 3 Characterization of the CT state in the bilayers. $\mathbf{a}, \mathbf{b}$ EQE spectra of the sub-bandgap absorption and the corresponding EL spectra for face-on (a) and edge-on (b) bilayers. Dashed lines are fits to the EQE using Eqs. 1 and 2. Fit parameters and their respective s.d. errors are reported in the figures. c Temperature-dependent $V_{O C}$ at $1,0.5$, and 0.1 suns, extrapolated to $\mathrm{OK}$ represented on the axis with the corresponding standard deviations

orientations of the donor layer. The $V_{\mathrm{OC}}$, on the other hand, is a substantial $150 \mathrm{mV}$ larger when the donor molecules are face-on compared to edge-on.

The solar cell characteristics here are averages of over 150 devices of each orientation; histograms for each parameter are available in Supplementary Fig. 5.
Open circuit voltage. There are several factors that can account for the change in the $V_{\mathrm{OC}}$, and we will explore each in turn: IP and $E_{\mathrm{CT}}$, radiative recombination, and non-radiative recombination.

A number of studies have demonstrated that changing molecular orientation in a film can lead to differences in the material energy levels ${ }^{7-9}, 11,16,17$, which can have a direct effect on the $V_{\text {OC. }}$. Indeed, ultraviolet photoelectron spectroscopy (UPS) measurements of neat p-SIDT $\left(\mathrm{FBTTh}_{2}\right)_{2}$ films show an increase on the order of $60 \mathrm{meV}$ in the IP of p-SIDT $\left(\mathrm{FBTTh}_{2}\right)_{2}$ when it is face-on (Supplementary Fig. 6). In good agreement, electronicstructure calculations performed on p-SIDT $\left(\mathrm{FBTTh}_{2}\right)_{2}$ also found that the face-on orientation has a deeper ionization potential (Supplementary Fig. 7). However, as the $V_{\text {OC }}$ varies by $150 \mathrm{mV}$, changing the molecular orientation has altered more than just the IP value.

It has been demonstrated numerous times that $E_{\mathrm{CT}}$ and $V_{\mathrm{OC}}$ tend to correlate according to: $E_{\mathrm{CT}}-q V_{\mathrm{OC}}=0.6 \pm 0.1 \mathrm{eV}^{23,46}$. The CT state is routinely studied with highly sensitive absorption techniques (here, we use external quantum efficiency, EQE), where it is identified as a shoulder at sub-bandgap energies ${ }^{46}$. The CT state can also be studied by emission spectra (here we use electroluminescence, EL), where it is identified as a featureless emission spectrum at low energies ${ }^{47}$. The $E_{\mathrm{CT}}$, located at the midpoint between the absorption and emission of the CT state, can be determined by a simultaneous fit to the measured absorption (Eq. 1) and emission spectra (Eq. 2) ${ }^{48}$ :

$$
\begin{gathered}
\sigma(E) E=\frac{f}{\sqrt{4 \pi \lambda k T}} \exp \left(\frac{-\left(E_{\mathrm{CT}}+\lambda-E\right)^{2}}{4 \lambda k T}\right) \\
\frac{I(E)}{E}=\frac{f}{\sqrt{4 \pi \lambda k T}} \exp \left(\frac{-\left(E_{\mathrm{CT}}-\lambda-E\right)^{2}}{4 \lambda k T}\right)
\end{gathered}
$$

In these equations, $k$ denotes Boltzmann's constant; $T$, temperature; and $E$, photon energy. The fit parameters are $E_{\mathrm{CT}}$ (energy of the CT state), $\lambda$ (reorganization energy), and $f$ (a parameter proportional to the number of CT states and the square of their coupling matrix element with the GS). Eqs. 1 and 2 are called the reduced absorption and emission spectra, due to the multiplication and division of the spectra by $E$, respectively.

Using Eqs. 1 and 2, fit simultaneously to the EQE and EL spectra, we obtain that $E_{\mathrm{CT}}$ is $1.38 \pm 0.02 \mathrm{eV}$ when p-SIDT $\left(\text { FBTTh }_{2}\right)_{2}$ is face-on vs. $1.32 \pm 0.03 \mathrm{eV}$ for the edge-on orientation. The EQE, EL and their corresponding fits are shown in Fig. 3a-b. The $60 \mathrm{meV}$ higher $E_{\mathrm{CT}}$ is in excellent agreement with the higher ionization energy of the face-on p-SIDT $\left(\mathrm{FBTTh}_{2}\right)_{2}$ layer, implying vacuum alignment at the interface to $\mathrm{C}_{60}$. However, it does not explain the full difference in $V_{\mathrm{OC}}$ upon changing molecular orientation.

Another estimate for the $E_{\mathrm{CT}}$ can be obtained by temperaturedependent $V_{\mathrm{OC}}$ measurements extrapolated to $0 \mathrm{~K}^{48,49}$, where the deviation from $E_{\mathrm{CT}}$ to $V_{\mathrm{OC}}$ at room temperature (RT) should correlate with losses in the solar cell. One model that has been demonstrated on a number of systems separates the losses from $E_{\mathrm{CT}}$ into radiative and non-radiative recombination, shown in Eq. $3^{48}$ :

$$
V_{\mathrm{OC}}=\frac{E_{\mathrm{CT}}}{q}-\Delta V_{\mathrm{rad}}(T)-\Delta V_{\text {nonrad }}(T)
$$

with $\Delta V_{\text {rad }}(T)$ and $\Delta V_{\text {nonrad }}(T)$ the temperature-dependent radiative and non-radiative recombination losses given by Eqs. 4 
Table 2 Summary of $E_{\mathrm{CT}}$ and recombination voltage losses for the bilayer solar cells

\begin{tabular}{|c|c|c|c|c|c|}
\hline Edge-on & $1.32 \pm 0.03$ & $197 \pm 4$ & $382 \pm 28$ & 0.74 & $0.69 \pm 0.04$ \\
\hline
\end{tabular}

and 5:

$$
\begin{gathered}
\Delta V_{\mathrm{rad}}(T)=-\frac{k T}{q} \ln \left(\frac{J_{\mathrm{SC}} h^{3} c^{2}}{f q 2 \pi\left(E_{\mathrm{CT}}-\lambda\right)}\right) \\
\Delta V_{\text {nonrad }}(T)=-\frac{k T}{q} \ln \left(\mathrm{EQE}_{\mathrm{EL}}\right)
\end{gathered}
$$

In these equations, $J_{\mathrm{SC}}$ represents the short circuit current; $h$, Planck's constant; $c$, speed of light; $E_{\mathrm{CT}}, f, \lambda$ are fit parameters from Eqs. 1 and 2 (Fig. 3a, b); and $\mathrm{EQE}_{\mathrm{EL}}$ is the total external quantum efficiency of electroluminescence.

The $V_{\text {OC }}$ values of each bilayer were measured at different light intensities and temperatures ranging from 190 to $310 \mathrm{~K}$, and extrapolated to $0 \mathrm{~K}$, as shown in Fig. 3c. For all light intensities the $V_{\mathrm{OC}}$ extrapolated to $0 \mathrm{~K}$ is $1.40 \pm 0.02 \mathrm{~V}$ for the face-on compared to $1.31 \pm 0.02 \mathrm{~V}$ for the edge-on solar cells, which is in close agreement to the $E_{\mathrm{CT}}$ values obtained from the fits of the EQE and EL spectra. This confirms our interpretation from above that the difference in $V_{\mathrm{OC}}$ is not solely due to energetics. In fact, from the temperature-dependent slopes in Fig. $3 c$, it is evident that the overall $V_{\text {OC }}$ loss is smaller in face-on than in edge-on bilayers.

As shown by Eq. 3, the voltage loss can be quantified into radiative and non-radiative recombination contributions, as has been outlined by $\mathrm{Rau}^{31}$ and Vandewal et al. ${ }^{48}$ Radiative recombination can be estimated according to Eq. 4 . We find that at room temperature $\Delta V_{\text {rad }}$ is very similar, $199 \pm 2 \mathrm{mV}$ and 197 $\pm 4 \mathrm{mV}$ for the face-on and edge-on bilayers, respectively, leading to the conclusion that losses due to radiative recombination are not a function of molecular orientation.

Non-radiative recombination remains poorly understood, yet it is considered to be among the primary reasons that the overall $V_{\text {OC }}$ loss has not decreased significantly in recent years ${ }^{25}$. Most organic blends reported in the literature lose 300-400 $\mathrm{mV}$ to nonradiative recombination, constituting $60 \%$ or more of the lost potential $^{25,48}$. To estimate the effect of orientation on nonradiative recombination, we refer to $\mathrm{EQE}_{\mathrm{EL}}$, defined as photons emitted per electrons injected into the device. The lower the radiative efficiency, the more non-radiative decay channels contribute to the overall recombination. Equation 5 relates $\mathrm{EQE}_{\mathrm{EL}}$ to the voltage loss.

Importantly, the measured $\mathrm{EQE}_{\mathrm{EL}}$ values differ by more than an order of magnitude between the two samples, with $3.2 \times 10^{-6}$ $\pm 1.4 \times 10^{-6}$ for the face-on and $2.3 \times 10^{-7} \pm 3.5 \times 10^{-7}$ for the edge-on bilayers. Using Eq. 5, we find that voltage losses due to non-radiative recombination are $316 \pm 10 \mathrm{mV}$ and $382 \pm 28 \mathrm{mV}$ for the face-on and edge-on solar cells, respectively.

Table 2 summarizes the energetic and recombination differences between the bilayers with the two orientations. The estimated difference in $V_{\mathrm{OC}}$ between the two orientations, $V_{\mathrm{OC} \text {,calc, }}$ calculated from the measured $E_{\mathrm{CT}}$ and the estimated losses due to radiative and non-radiative recombinations (Eq. 3) is in very good agreement with the measured $\Delta V_{\mathrm{OC}}$, determined experimentally from the $J-V$ characteristics.

The significance of these findings lies in the differences in nonradiative recombination: on average, the edge-on solar cells lose
$66 \mathrm{mV}$ more voltage than face-on cells due to non-radiative recombination. Using the same system but flipping the donor molecular orientation, the non-radiative recombination pathway has been altered, implying it is sensitive to the molecular alignment at the donor/acceptor interface. Interestingly, these experimental results are fully consistent with a recent theoretical study: Chen et al. ${ }^{3}$ found that the higher $E_{\mathrm{CT}}$ value and greater hole delocalization and migration away from a face-on pentacene/ $\mathrm{C}_{60}$ interface caused a decrease in vibronic coupling of the CT state to the GS, thus reducing the non-radiative recombination rate. While the results by Chen et al. ${ }^{3}$ refer to model molecular packings of pentacene, the similarity to our findings on p-SIDT $\left(\mathrm{FBTTh}_{2}\right)_{2} / \mathrm{C}_{60}$, namely a smaller $E_{\mathrm{CT}}$ and more non-radiative recombination in the edge-on bilayers, is striking.

Short circuit current. In contrast to the $V_{\mathrm{OC}}$, the $J_{\mathrm{SC}}$ appears independent of molecular orientation (Fig. 1). However, due to alignment of the molecular transition dipoles, the absorption strength of the two bilayers toward normal incident light is significantly different: face-on p-SIDT $\left(\mathrm{FBTTh}_{2}\right)_{2}$ films have a two times higher absorbance as edge-on films (Supplementary Fig. 8). Using a combination of Monte Carlo (MC) and molecular dynamics (MD) simulations, we find that $\mathrm{p}-\operatorname{SIDT}\left(\mathrm{FBTTh}_{2}\right)_{2}$ has a unique unit cell, shown in Supplementary Fig. 9, true for both molecular orientations. As a consequence, half the p-SIDT $\left(\text { FBTTh }_{2}\right)_{2}$ molecules in the edge-on orientation are lying down with their long edge parallel to the substrate, and half the molecules are standing up on their end, with their sides perpendicular to the substrate (as depicted in Fig. 1a). In the face-on orientation, all the p-SIDT $\left(\mathrm{FBTTh}_{2}\right)_{2}$ molecules are parallel to the substrate. This model for the packing of p-SIDT $\left(\mathrm{FBTTh}_{2}\right)_{2}$ is in good agreement with the two-times higher absorbance of the face-on films. Furthermore, to verify that the results we have reported thus far are not a consequence of the different geometry of molecular transition dipoles with respect to light, we measured the current-voltage $(J-V)$ curves for the bilayer solar cells as a function of illumination angle. The $V_{\mathrm{OC}}$ remains unchanged, while the $J_{\mathrm{SC}}$ increases by a small amount (Supplementary Fig. 10).

The similar $J_{S C}$ values are in agreement with the EQE (quantum efficiency per incident photon) of the bilayers, both peaking at about $25 \%$ on average. However, when the EQE spectra are corrected for absorption of the active layer (device absorption corrected for parasitic absorption, more detail in Supplementary Fig. 11), we obtain quantum efficiency spectra per absorbed photons, i.e., IQE. EQE and IQE spectra of the bilayers are shown in Fig. 4a. For reference, the unitless absorption spectra of p-SIDT $\left(\mathrm{FBTTh}_{2}\right)_{2}$ and $\mathrm{C}_{60}$ are shown in the background.

IQE of the edge-on bilayers is higher than the face-on bilayers (Fig. 4a). A higher IQE indicates that in edge-on bilayers fewer excitons and charges recombine at short circuit. In other words, the edge-on solar cells have more efficient charge generation. Charge generation depends on exciton quenching efficiency and geminate recombination. Photoluminescence (PL) quenching measurements comparing neat edge-on and face-on films with their respective bilayer structure show that both molecular orientations have the same quenching efficiency (Supplementary Fig. 12). Therefore, the difference in the IQE values must 
originate from geminate recombination after charge transfer across the heterojunction. This finding is consistent with the results of our electronic-structure calculations, which indicate that electronic coupling between CT state and GS is weaker in the edge-on configuration than in the face-on configuration
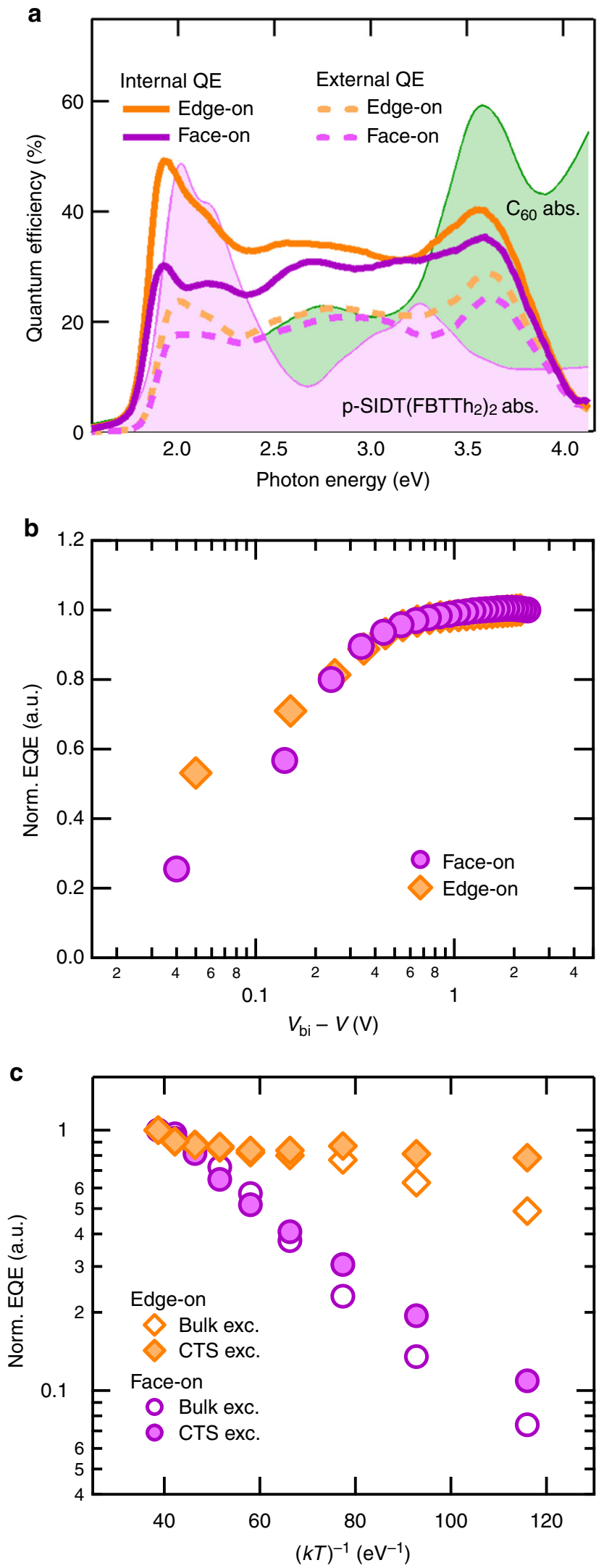

(Supplementary Fig. 13). A smaller electronic coupling is expected to decrease the rate of geminate recombination as the CT state tends to dissociate, shifting the generation-recombination balance towards free charge formation, as seen here ${ }^{2}$.

For a deeper understanding of the differences in IQE, we measured the EQE under varying electric fields and temperatures. These measurements are complex, since they reflect the combination of many processes such as exciton diffusion, charge transfer, charge generation, bimolecular recombination, and charge transport. However, with the appropriate conditions and analysis, EQE measurements can be used to gain insight into charge generation/geminate recombination. Specifically, to eliminate effects of exciton diffusion and charge transfer, EQE spectra can be analyzed at energies corresponding to CT state absorption. Also, since these devices are bilayers and the measurements are carried out at low light intensities and under an internal field ( $J_{\mathrm{SC}}$ conditions, unless otherwise stated), bimolecular recombination and the effect of differences in of charge transport are expected to be negligible. Under these conditions, our EQE measurements should reflect the dependence of charge generation/geminate recombination on electric field and temperature.

First, we asked if changing interfacial molecular orientation has an impact on the Coulomb binding energy of the CT state. The binding energy, a consequence of the electrostatic attraction between opposite charge carriers, depends on the electron-hole separation and the dielectric constant ${ }^{50}$. The binding energy can be overcome with the assistance of a field ${ }^{40,51}$, and thus it may follow that the field-dependence of generation would be different for the two bilayers ${ }^{52}$. Figure $4 \mathrm{~b}$ shows the effect of an electric field on the EQE of the devices: both have a very similar dependence on the electric field. The EQE values in Fig. $4 \mathrm{~b}$ are normalized to the efficiency at the strongest applied bias, and the field across the device is corrected by the built-in voltage, analogous to photocurrent analysis (for bias-dependent EQE spectra, see Supplementary Fig. 14). Time delayed collection field (TDCF) measurements confirm no significant differences in the field dependence of generation between the two orientations (Supplementary Fig. 15). Furthermore, by TDCF there is no difference in field-dependence of generation for excitations at $350 \mathrm{~nm}, 600 \mathrm{~nm}$, or $650 \mathrm{~nm}$, ruling out any effects of hot-exciton generation. All these results indicate that the binding energy of the CT state is not a function of molecular orientation.

Next, we turned our attention to the temperature-dependence of charge generation. Figure $4 \mathrm{c}$ shows EQE values normalized to the EQE at RT, as a function of temperature (for temperaturedependent EQE spectra, see Supplementary Fig. 16). The temperature dependence is shown for absorption over all energies, as well as absorption corresponding only to the CT state $(\sim 1.2-1.5 \mathrm{eV})$. Overall, while charge generation in both bilayers is temperature-dependent, the temperature-dependence in the face-on bilayers is much stronger, indicating a larger activation energy for charge generation. To illustrate this point, if we make crude simplifications and extrapolate the EQE values to the limit of temperature $\rightarrow 0 \mathrm{~K}$, we find that generation in the faceon bilayer becomes negligible, while the edge-on bilayer can still

Fig. 4 Efficiency of charge generation. a EQE (dashed lines) and IQE (continuous lines) spectra of bilayer solar cells with varying orientation. The absorption spectra of p-SIDT(FBTTh $)_{2}$ and $\mathrm{C}_{60}$ are plotted in the background for reference. $\mathbf{b}$ Bias-dependent $\mathrm{EQE}$ values corresponding to $\mathrm{p}$-SIDT $\left(\mathrm{FBTTh} \mathrm{C}_{2}\right)_{2}$ absorption, integrated and normalized to the value under the highest field. The applied bias was corrected for the built-in potential. c Temperature-dependent EQE values, integrated and normalized to the value at room temperature. Open symbols are EQE values integrated over bulk absorption, and full symbols are EQE values integrated only for CT state absorption 
generate about 10-40\% carriers (as compared to carrier generation at RT). Charge generation resulting from bulk and direct CT state excitations follows similar temperature dependences, as a function of $\mathrm{p}$-SIDT $\left(\mathrm{FBTTh}_{2}\right)_{2}$ orientation. The contrast in temperature dependence is therefore not due to differences in exciton diffusion or electron transfer, but is instead a result of difference in charge generation with interfacial molecular orientation. This can be explained by a larger barrier to charge generation in the face-on bilayer due to elements such as electronic coupling between the CT states and separated states or polarization at the donor/acceptor interface.

\section{Discussion}

In conclusion, we have been able to fabricate donor-acceptor bilayers with sharp interfaces and well-defined p-SIDT $\left(\mathrm{FBTTh}_{2}\right)_{2}$ molecular orientations: either face-on or edge-on with respect to the substrate. These orientations are preserved through the donor film to the interface with $\mathrm{C}_{60}$, with none-to-minimal diffusion at the donor/acceptor interface. This unprecedented precise morphological control reveals the genuine effects of molecular orientation on photovoltaic performance. Edge-on p-SIDT $\left(\mathrm{FBTTh}_{2}\right)_{2}$ bilayers suffer from greater non-radiative recombination and a reduced $E_{\mathrm{CT}}$, which result in a substantial $V_{\mathrm{OC}}$ loss of $150 \mathrm{mV}$. However, charge generation is more efficient when the donor/acceptor interface is edge-on, evidenced by a higher IQE. This is attributed to reduced CT state-GS electronic coupling as well as smaller activation energy for charge generation in the edge-on bilayer, which may be a consequence of a reduced barrier between CT state and charge-separated states or favorable polarization at the donor/acceptor interface.

The lessons learned from p-SIDT(FBTTh $\left.{ }_{2}\right)_{2} / \mathrm{C}_{60}$ bilayers can be summarized into two major points: First, in applications which benefit from high radiative efficiency (such as LEDs and OPVs), interfacial molecular orientation should reduce non-radiative recombination (by reducing charge recombination through triplet states or through vibronic coupling of the CT state to the GS). Our results establish that face-on molecular orientations would achieve higher radiative efficiency. Second, In applications where charge separation is important (such as photodetectors and OPVs), the electronic couplings between the CT state and the GS, as well as the activation energy for charge generation should both be minimized. This can perhaps be accomplished by beneficial polarization effects. Thus, our results determine that interfacial molecular orientation should be edge-on for intrinsic, efficient charge generation.

Overall, in the case of OPV, these two lessons go in opposite directions. These results highlight that to achieve high performance in OPV, the electronic coupling for face-on donor/ acceptor interactions must be reduced to eliminate geminate recombination. Conversely, more research on non-radiative recombination is necessary in order to curtail the resulting losses and benefit from the improved charge generation in an edgeon donor/acceptor interaction. It may be possible to tackle both problems from the perspectives of molecular design and clever device engineering.

\footnotetext{
Methods

Sample preparation. p-SIDT(FBTTh $\left.{ }_{2}\right)_{2}$ (benzo[1,2-b:4,5-b]bis(4,4'-dihexyl- $4 H$ silolo[3,2- $b$ ] thiophene-2,2'-diyl)bis(6-fluoro-4-(5'-hexyl-[2,2'-bithiophene]-5-yl) benzo[c] $[1,2,5]$ thiadiazole) was synthesized according to the previously reported scheme $^{45} \cdot \mathrm{p}$-SIDT(FBTTh $)_{2}$ was dissolved at a concentration of $15 \mathrm{mg} \mathrm{m}^{-1}$ in pristine $\mathrm{CB}$, or $\mathrm{CB}$ with $0.4 \%$ DIO v/v. In the $\mathrm{CB}+\mathrm{DIO}$ solution, the conditions correspond to a ratio of two molecules DIO for every one molecule of p-SIDT $\left(\text { FBTTh }_{2}\right)_{2}{ }^{53}$. The face-on films used in this study were achieved by spin-casting p-SIDT $\left(\text { FBTTh }{ }_{2}\right)_{2}$ from pristine CB solutions, and the edge-on films were achieved by casting from $\mathrm{CB}+\mathrm{DIO}$ solutions, at 2000 round per minute (RPM). Bilayer devices were fabricated on glass substrates sputtered with ITO, and coated with 35
}

$\mathrm{nm}$ poly(3,4-ehtylenedioxythiophene):poly(styrenesulfonate) (PEDOT:PSS). A $\mathrm{C}_{60}$ layer was thermally evaporated on p-SIDT(FBTTh ${ }_{2}$, followed by bathocuproine $(\mathrm{BCP})$ and aluminum $(\mathrm{Al})$. The final device structure for nearly all measurements reported herein is as follows: ITO/PEDOT $(35 \mathrm{~nm}) / \mathrm{p}$-SIDT $\left(\mathrm{FBTTh}_{2}\right)_{2}(45 \mathrm{~nm}) / \mathrm{C}_{60}$ $(45 \mathrm{~nm}) / \mathrm{BCP}(4 \mathrm{~nm}) / \mathrm{Al}(80 \mathrm{~nm})$

GIWAXS analysis. GIWAXS was performed at Stanford Synchrotron Radiation Lightsource beamline 11-3 with a MAR345 image plate. The data was calibrated and reduced using WxDiff software package ${ }^{54}$. To characterize the amount of edgeon vs. face-on material, a cake slice around the $\pi$-stacking peak, between $Q=1.8 \AA^{-1}$ and $2.0 \AA^{-1}$, was reduced to a pole figure. An adjacent cake slice between $Q=1.7 \AA^{-1}$ and $1.8 \AA^{-1}$ was subtracted from the data to account for background scattering (see Supplementary Fig. 1). The data was fit to two in-plane $\pi$-stacking peaks at about $-90^{\circ}$ and $+90^{\circ}$, one out-of-plane $\pi$-stacking peak at $0^{\circ}$, and four peaks around $\pm 30^{\circ}$ and $\pm 55^{\circ}$ representing the $\mathrm{SiO}_{2}$ substrate background. The out-of-plane peak area was compared to the average of the two in-plane peak areas to arrive at the face-on to edge-on ratio for each sample. Samples for GIWAXS were prepared on cleaned $\mathrm{SiO}_{2}$ substrates, coated with PEDOT:PSS and p-SIDT(FBTTh $\left.{ }_{2}\right)_{2}$ cast from $15 \mathrm{mg} \mathrm{ml}^{-1} \mathrm{CB}$ or CB+DIO

The $\mathrm{C}_{60}$ scattering as a function of $\mathrm{C}_{60}$ thickness was tracked by reducing cake slices shown in Supplementary Fig. 3 to $I$ vs. Q. We selected these specific cake slices for analysis because they contain non-overlapping peaks from both the small molecule and the $\mathrm{C}_{60}$, allowing a simultaneous comparison of contributions from both materials. This data was fit to a linear combination of neat p-SIDT $\left(\mathrm{FBTTh}_{2}\right)_{2}$ and neat $\mathrm{C}_{60}$ data, and the fit coefficient for $\mathrm{C}_{60}$ was reported. Fits are shown in Supplementary Fig. 3 along with the neat data. Fits have some discrepancy due to small changes in peak shape but are reasonably accurate in depicting overall trends Samples for these measurements were prepared on cleaned $\mathrm{SiO}_{2}$ substrates, coated with PEDOT:PSS and p-SIDT(FBTTh $)_{2}$ cast from $15 \mathrm{mg} \mathrm{ml}^{-1} \mathrm{CB}$ or $\mathrm{CB}+\mathrm{DIO}$, with $\mathrm{C}_{60}$ evaporated for thicknesses of $0-30 \mathrm{~nm}$.

Ultraviolet photoelectron spectroscopy. UPS samples were prepared on ITOsputtered glass substrates cleaned by sonication in water, acetone, and isopropyl alcohol for $15 \mathrm{~min}$, followed by UVO treatment for $1 \mathrm{~h}$. ITO pieces were immediately transferred to the glove box. Solutions of $7 \mathrm{mg} \mathrm{ml}^{-1} \mathrm{p}$-SIDT $\left(\mathrm{FBTTh}_{2}\right)_{2}$ in $\mathrm{CB}$ or $\mathrm{CB}+0.4 \%$ DIO were prepared ahead of time, and allowed to stir on the hot plate at $110^{\circ} \mathrm{C}$. Prior to casting the films, the solutions were brought to room temperature. Films were cast at 4000 RPM for film thicknesses of about $10 \mathrm{~nm}$; thicker films of p-SIDT( $\left(\mathrm{FBTTh}_{2}\right)_{2}$ led to distortion of the UPS signal due to charging in the film. The UPS samples were transferred to an ultrahigh vacuum chamber from the nitrogen glove box in an airtight chamber to avoid any surface degradation or contamination with air. The UPS was measured with a HeI source $(21.2 \mathrm{eV})$, at pressures of low $10^{-7} \mathrm{~Pa}$, a constant pass of $5 \mathrm{eV}$, current of $10 \mathrm{~mA}$, and the anode set to $6 \mathrm{kV}$. The ionization potential was calculated according to $I_{\mathrm{p}}=21.2 \mathrm{eV}-\left(E_{\text {cutoff }}-E_{\mathrm{g}}\right)^{55}$, where the $E_{\text {cutoff }}$ and $E_{\mathrm{g}}$ were determined by the onset at high and low binding energies, respectively. The energy resolution of the UPS measurements is below $10 \mathrm{meV}$.

J-V characteristics. Solar-cell device properties were measured under illumination by a simulated $100 \mathrm{~mW} \mathrm{~cm} \mathrm{~cm}^{-2}$ AM1.5 G light source using a $300 \mathrm{~W}$ Xe arc lamp with an AM 1.5 global filter. The irradiance was adjusted to one sun with a standard silicon photovoltaic calibrated by the National Renewable Energy Laboratory. Temperature dependent $V_{\mathrm{OC}}$ in the range of $275-300 \mathrm{~K}$ was collected using a custom Peltier cooled sample holder under illumination from the above described light source in combination with optical density filters to reduce the intensity. In the range of temperatures below $275 \mathrm{~K}$, a helium cryostat was used for temperature control and illumination was achieved with a high power $1 \mathrm{~W}, 445 \mathrm{~nm}$ laser diode (fluence was tuned with the DC bias applied to the laser diode to match light intensity used in the solar simulator). Bias dependent $J-V$ spectra were measured by changing the angle of the light source, while keeping the device constant. Before each $J-V$ measurement light intensity was calibrated to 1 -sun using the calibrated silicon photovoltaic.

TEM and cross-sectional TEM. TEM samples were prepared by casting a layer of p-SIDT $\left(\text { FBTTh }{ }_{2}\right)_{2}$ from CB or CB+DIO on PEDOT:PSS, and floating pieces of the film on deionized (DI) water. Film pieces were transferred to TEM grids and allowed to dry overnight. High-resolution images were taken with an FEI Titan FEG High Resolution microscope. The TEM images were collected using a lowdose electron beam (spot size 6) to avoid beam damage, and a small defocus to enhance the contrast in the images.

Using an FEI focused ion-beam (FIB) microscope, a $20 \mu \mathrm{m}$ long slice with thickness of about $200 \mathrm{~nm}$ was cut from a bilayer device (prepared as described above), and mounted on a TEM grid. The donor/acceptor interface in the bilayers was then imaged by HR-TEM. The procedure followed has been described in detail previously $y^{56,57}$. Careful attention was devoted to minimize exposure of the sample to high-energy electron and ion beams, thereby reducing damage as much as possible

Atomic force microscopy. Atomic force microscopy (AFM) images were collected in air, using a Si tip, and an Innova AFM operated under tapping mode. 
UV-visible absorption spectroscopy. Absorption spectra of neat face-on and edge-on p-SIDT(FBTTh $\left.{ }_{2}\right)_{2}$ and $\mathrm{C}_{60}$ films were collected using a Perkin Elmer Lambda 750 spectrophotometer. Samples for absorption measurements were prepared on clean glass slides, following the same procedure used to prepare the respective layer in the bilayer solar cell devices: face-on and edge-on p-SIDT $\left(\mathrm{FBTTh}_{2}\right)_{2}$ films were solution cast from their respective solutions, and $\mathrm{C}_{60}$ films were thermally evaporated. The film thickness for all samples was $45 \mathrm{~nm}$.

External and internal quantum efficiency. EQE characteristics were measured in a nitrogen-filled glovebox using a setup at UCSB and in Potsdam University. At UCSB, the EQE setup consisted of a 75 W Xe light source, monochromator, optical chopper, lock-in amplifier, and a National Institute of Standards and Technology calibrated silicon photodiode for power-density calibration. At Potsdam University, the EQE setup is similar, but used a $200 \mathrm{~W}$ halogen lamp (Philips), and a UV enhanced silicon photodiode to calibrate the visible spectra, or a germanium photodiode to calibrate the near infrared spectra. Both photodiodes were calibrated by Newport. For the sub-bandgap EQE, higher sensitivity settings were used with a longer time delay between measurement points. Bias-dependent $\mathrm{EQE}$ was collected on the setup in Potsdam University, coupled to a Keithley source-measure-unit used to apply a bias while the EQE spectra were recorded. Temperature-dependent EQE measurements were collected with a setup at UCSB, following a similar procedure, using a nitrogen-cooled cryostat.

Total absorption of solar cell devices was measured with an integrating sphere, and corrected for parasitic absorption as determined for the bilayers using a transfer matrix model ${ }^{58}$. Subtracting the parasitic absorption from the total device absorption then gives the active layer absorption, and dividing EQE spectra by the corresponding active layer absorption gives the IQE spectra of the device.

EL and its efficiency. EL spectra for the bilayers were collected directly from the solar cell devices, by applying a bias that is close to the turn-on voltage of the devices. The resulting emission was collected with an Andor SR393i-B spectrometer provided with a cooled silicon detector DU420ABR-DD and a cooled InGaAs DU491A-1.7 detector. The spectra were corrected for detector response using a blackbody spectrum.

The EL efficiency was collected by applying a small bias to the bilayer devices, and placing a calibrated silicon photodiode directly in front of the device to collect the resulting emission. The angle between the calibrated silicon photodiode and the device was varied to account for anisotropy in emission intensity.

PL quenching measurements. PL samples were prepared by spin-coating p-SIDT $\left(\mathrm{FBTTh}_{2}\right)_{2}$ films (face-on or edge-on) on clean glass slides, and evaporating $\mathrm{C}_{60}$ on half the slides, analogous to the preparation of the active layer in the bilayer devices. The samples were encapsulated inside the glovebox by applying epoxy around the edges of the glass slide and putting another glass slide on top, to create an air-tight, nitrogen environment. The samples were excited by a laser excitation at $455 \mathrm{~nm}$, and the PL collected using a silicon CCD camera, cooled to $-70^{\circ} \mathrm{C}$. A series of lenses were used to collect the PL and focus it on the CCD camera slit. The resulting spectra were corrected for instrument response by using a reference blackbody spectrum.

Time-delayed collection field measurements. Excitation is realized by a laser system consisting of a Libra USP-1K-HE with a pulse energy of $\sim 4.0 \mathrm{~mJ}$ at $1 \mathrm{kHz}$ and an OPerA Solo for wavelength selection. The pre- and collection voltage is applied via an Agilent $81150 \mathrm{~A}$ pulse generator in combination with a home-built amplifier. Currents through the devices are measured via a $50 \Omega$ resistor and recorded with an Yokogawa DL9140 oscilloscope.

Electronic-structure calculations. The p-SIDT $\left(\mathrm{FBTTh}_{2}\right)_{2}$ bulk structure and $\mathrm{C}_{60} / \mathrm{p}$-SIDT(FBTTh $\left.{ }_{2}\right)_{2}$ bilayer structure were generated by a combination of MC and MD simulations. The MD simulations were run for $200 \mathrm{ps}$ at $300 \mathrm{~K}$ with under the NVT ensemble using the Verlet integrator with a time step of $1 \mathrm{fs}$. The temperature was maintained by the Nose-Hoover thermostat. A spherical cutoff of $1.25 \mathrm{~nm}$ for the summation of van der Waals interactions and the Ewald solver for long-range Coulomb interactions was used throughout. The COMPASS force field as implemented in the Forcite program of Materials Studio was used for the MD simulation $s^{59}$. Density-functional theory calculations using the range-separated HSE functional were then carried out for face-on and edge-on p-SIDT( $\left.\mathrm{FBTTh}_{2}\right)_{2}$ slabs under periodic boundary conditions using the plane-wave based Vienna Ab-initio Simulation Package ${ }^{60-63}$.

The electron couplings between diabatic CT state and the GS of the p-SIDT $\left(\mathrm{FBTTh}_{2}\right)_{2} / \mathrm{C}_{60}$ complexes were evaluated by means of the generalized MullikenHush approach at the MD generated geometry. These calculations were performed with the B3LYP functional and 6-31G $(\mathrm{d}, \mathrm{p})$ basis set, using the Q-Chem package ${ }^{64}$.

Data availability. Data and materials necessary to replicate this work are available upon request from the authors.
Received: 1 November 2016 Accepted: 31 May 2017

Published online: 19 July 2017

\section{References}

1. Rand, B. P. et al. The impact of molecular orientation on the photovoltaic properties of a Phthalocyanine/Fullerene Heterojunction. Adv. Funct. Mater 22, 2987-2995 (2012).

2. Yi, Y., Coropceanu, V. \& Brédas, J.-L. Exciton-dissociation and chargerecombination processes in pentacene/C60 solar cells: Theoretical insight into the impact of interface geometry. J. Am. Chem. Soc. 131, 15777-15783 (2009).

3. Chen, X.-K., Ravva, M. K., Li, H., Ryno, S. M. \& Brédas, J.-L. Effect of molecular packing and charge delocalization on the nonradiative recombination of charge-transfer states in organic solar cells. Adv. Energy Mater. 6, 1601325 (2016).

4. Yang, B. et al. Impact of electron delocalization on the nature of the chargetransfer states in model Pentacene/c60 interfaces: A density functional theory study. J. Phys. Chem. C 118, 27648-27656 (2014).

5. Verlaak, S. et al. Electronic structure and geminate pair energetics at organic-organic interfaces: The case of Pentacene/C60 Heterojunctions. Adv. Funct. Mater. 19, 3809-3814 (2009).

6. Idé, J. et al. Interfacial dipole and band bending in model pentacene/C60 heterojunctions. Int. J. Quantum Chem. 113, 580-584 (2013).

7. Tumbleston, J. R. et al. The influence of molecular orientation on organic bulk heterojunction solar cells. Nat. Photonics 8, 385-391 (2014).

8. Zhang, L., Roy, S. S., Hamers, R. J., Arnold, M. S. \& Andrew, T. L. Molecular orientation-dependent interfacial energetics and built-in voltage tuned by a template graphene monolayer. J. Phys. Chem. C 119, 45-54 (2014).

9. Chen, W., Qi, D.-C., Huang, H., Gao, X. \& Wee, A. T. S. Organic-organic heterojunction interfaces: Effect of molecular orientation. Adv. Funct. Mater. 21, 410-424 (2011).

10. Alloway, D. M. \& Armstrong, N. R. Organic heterojunctions of layered perylene and phthalocyanine dyes: characterization with UV-photoelectron spectroscopy and luminescence quenching. Appl. Phys. A 95, 209-218 (2009).

11. Kitchen, B. et al. Tuning open-circuit voltage in organic solar cells with molecular orientation. ACS Appl. Mater. Interfaces 7, 13208-13216 (2015).

12. Ojala, A. et al. Merocyanine/C60 planar heterojunction solar cells: Effect of dye orientation on exciton dissociation and solar cell performance. Adv. Funct. Mater. 22, 86-96 (2012).

13. Graham, K. R. et al. Re-evaluating the role of sterics and electronic coupling in determining the open-circuit voltage of organic solar cells. Adv. Mater. 25, 6076-6082 (2013).

14. Ayzner, A. L., Nordlund, D., Kim, D.-H., Bao, Z. \& Toney, M. F. Ultrafast electron transfer at organic semiconductor interfaces: Importance of molecular orientation. J. Phys. Chem. Lett. 6, 6-12 (2015).

15. Hörmann, U. et al. Voc from a morphology point of view: The influence of molecular orientation on the open circuit voltage of organic planar heterojunction solar cells. J. Phys. Chem. C 118, 26462-26470 (2014)

16. Lin, H.-C. et al. Influence of molecular orientation on charge-transfer processes at phthalocyanine/metal oxide interfaces and relationship to organic photovoltaic performance. J. Phys. Chem. C 119, 10304-10313 (2015)

17. Duhm, S. et al. Orientation-dependent ionization energies and interface dipoles in ordered molecular assemblies. Nat. Mater. 7, 326-332 (2008).

18. Treat, N. D. et al. Interdiffusion of PCBM and P3HT reveals miscibility in a photovoltaically active blend. Adv. Energy Mater 1, 82-89 (2011).

19. Ngongang Ndjawa, G. O. et al. Impact of molecular orientation and spontaneous interfacial mixing on the performance of organic solar cells. Chem. Mater. 27, 5597-5604 (2015).

20. Zimmerman, J. D. et al. Independent control of bulk and interfacial morphologies of small molecular weight organic heterojunction solar cells. Nano Lett. 12, 4366-4371 (2012).

21. Zimmerman, J. D. et al. Control of interface order by inverse quasi-epitaxial growth of squaraine/fullerene thin film photovoltaics. ACS Nano 7, 9268-9275 (2013).

22. Deibel, C., Strobel, T. \& Dyakonov, V. Role of the charge transfer state in organic donor-acceptor solar cells. Adv. Mater. 22, 4097-4111 (2010).

23. Vandewal, K., Tvingstedt, K., Gadisa, A., Inganäs, O. \& Manca, J. V. On the origin of the open-circuit voltage of polymer-fullerene solar cells. Nat. Mater 8, 904-909 (2009).

24. Gruber, M. et al. Thermodynamic efficiency limit of molecular donor-acceptor solar cells and its application to diindenoperylene/C60-based planar heterojunction devices. Adv. Energy Mater. 2, 1100-1108 (2012).

25. Yao, J. et al. Quantifying losses in open-circuit voltage in solution-processable solar cells. Phys. Rev. Appl. 4, 014020 (2015).

26. Rau, U., Paetzold, U. W. \& Kirchartz, T. Thermodynamics of light management in photovoltaic devices. Phys. Rev. B 90, 035211 (2014).

27. Liu, J. et al. Fast charge separation in a non-fullerene organic solar cell with a small driving force. Nat. Energy 1, 16089 (2016). 
28. Bässler, H. \& Köhler, A. 'Hot or cold': how do charge transfer states at the donor-acceptor interface of an organic solar cell dissociate? Phys. Chem. Chem. Phys. 17, 28451-28462 (2015).

29. Gao, F. \& Inganäs, O. Charge generation in polymer-fullerene bulkheterojunction solar cells. Phys. Chem. Chem. Phys. 16, 20291-20304 (2014).

30. Few, S., Frost, J. M. \& Nelson, J. Models of charge pair generation in organic solar cells. Phys. Chem. Chem. Phys. 17, 2311-2325 (2014)

31. Rau, U. Reciprocity relation between photovoltaic quantum efficiency and electroluminescent emission of solar cells. Phys. Rev. B 76, 085303 (2007)

32. Miller, O. D., Yablonovitch, E. \& Kurtz, S. R. Strong internal and external luminescence as solar cells approach the Shockley-Queisser limit. IEEE J. Photovolt. 2, 303-311 (2012).

33. Faist, M. A. et al. Competition between the charge transfer state and the singlet states of donor or acceptor limiting the efficiency in polymer:Fullerene solar cells. J. Am. Chem. Soc. 134, 685-692 (2012).

34. Veldman, D., Meskers, S. C. J. \& Janssen, R. A. J. The energy of charge-transfer states in electron donor-acceptor blends: Insight into the energy losses in organic solar cells. Adv. Funct. Mater. 19, 1939-1948 (2009).

35. Bakulin, A. A. et al. The role of driving energy and delocalized states for charge separation in organic semiconductors. Science 335, 1340-1344 (2012).

36. Vithanage, D. A. et al. Visualizing charge separation in bulk heterojunction organic solar cells. Nat. Commun. 4, 2334 (2013).

37. Jamieson, F. C. et al. Fullerene crystallisation as a key driver of charge separation in polymer/fullerene bulk heterojunction solar cells. Chem. Sci. 3, 485-492 (2012).

38. Bernardo, B. et al. Delocalization and dielectric screening of charge transfer states in organic photovoltaic cells. Nat. Commun. 5, 3245 (2014).

39. Ward, A. J. et al. The impact of driving force on electron transfer rates in photovoltaic donor-acceptor blends. Adv. Mater. 27, 2496-2500 (2015).

40. Albrecht, S. et al. On the field dependence of free charge carrier generation and recombination in blends of $\mathrm{PCPDTBT} / \mathrm{PC}_{70} \mathrm{BM}$ : Influence of solvent additives. J. Phys. Chem. Lett. 3, 640-645 (2012).

41. Dibb, G. F. A., Jamieson, F. C., Maurano, A., Nelson, J. \& Durrant, J. R. Limits on the fill factor in organic photovoltaics: Distinguishing nongeminate and geminate recombination mechanisms. J. Phys. Chem. Lett. 4, 803-808 (2013)

42. Nayak, P. K., Narasimhan, K. L. \& Cahen, D. Separating charges at organic interfaces: Effects of disorder, hot states, and electric field. J. Phys. Chem. Lett. 4 1707-1717 (2013)

43. Bartelt, J. A. et al. The importance of fullerene percolation in the mixed regions of polymer-fullerene bulk heterojunction solar cells. Adv. Energy Mater. 3, 364-374 (2013)

44. Monahan, N. R., Williams, K. W., Kumar, B., Nuckolls, C. \& Zhu, X.-Y. Direct observation of entropy-driven electron-hole pair separation at an organic semiconductor interface. Phys. Rev. Lett. 114, 247003 (2015).

45. Love, J. A. et al. Silaindacenodithiophene-based molecular donor: Morphological features and use in the fabrication of compositionally tolerant, high-efficiency bulk heterojunction solar cells. J. Am. Chem. Soc. 136, 3597-3606 (2014)

46. Vandewal, K. et al. The relation between open-circuit voltage and the onset of photocurrent generation by charge-transfer absorption in polymer: Fullerene bulk heterojunction solar cells. Adv. Funct. Mater. 18, 2064-2070 (2008)

47. Tvingstedt, K. et al. Electroluminescence from charge transfer states in polymer solar cells. J. Am. Chem. Soc. 131, 11819-11824 (2009).

48. Vandewal, K., Tvingstedt, K., Gadisa, A., Inganäs, O. \& Manca, J. V. Relating the open-circuit voltage to interface molecular properties of donor:acceptor bulk heterojunction solar cells. Phys. Rev. B 81, 125204 (2010).

49. Hörmann, U. et al. Quantification of energy losses in organic solar cells from temperature-dependent device characteristics. Phys. Rev. B 88, 235307 (2013).

50. Köhler, A. \& Bässler, H. Electronic Processes in Organic Semiconductors (Wiley-VCH Verlag GmbH \& Co. KGaA, 2015).

51. Schweitzer, B., Arkhipov, V. I. \& Bässler, H. Field-induced delayed photoluminescence in a conjugated polymer. Chem. Phys. Lett. 304, 365-370 (1999).

52. Schwarz, C. et al. Role of the effective mass and interfacial dipoles on exciton dissociation in organic donor-acceptor solar cells. Phys. Rev. B 87, 155205 (2013).

53. Love, J. A. et al. Interplay of solvent additive concentration and active layer thickness on the performance of small molecule solar cells. Adv. Mater. 26, 7308-7316 (2014)

54. Mannsfeld, S. C. B., Tang, M. L. \& Bao, Z. Thin film structure of triisopropylsilylethynyl-functionalized pentacene and tetraceno[2,3-b] thiophene from Grazing incidence X-Ray diffraction. Adv. Mater. 23, 127-131 (2011).

55. Seo, J. H. \& Nguyen, T.-Q. Electronic properties of conjugated polyelectrolyte thin films. J. Am. Chem. Soc. 130, 10042-10043 (2008).

56. Moon, J. S. et al. Effect of processing additive on the nanomorphology of a bulk heterojunction material. Nano Lett. 10, 4005-4008 (2010).
57. Moon, J. S., Takacs, C. J., Sun, Y. \& Heeger, A. J. Spontaneous formation of bulk heterojunction nanostructures: Multiple routes to equivalent morphologies. Nano Lett. 11, 1036-1039 (2011).

58. Burkhard, G. F., Hoke, E. T. \& McGehee, M. D. Accounting for interference, scattering, and electrode absorption to make accurate internal quantum efficiency measurements in organic and other thin solar cells. Adv. Mater. 22, 3293-3297 (2010)

59. Materials Studio Program, version 6.1 (Accelrys Software Inc., San Diego, CA 2012).

60. Kresse, G. \& Furthmuller, J. Efficiency of Ab-Initio total energy calculations for metals and semiconductors using a plane-wave basis set. Comp. Mater. Sci. 6, 15-50 (1996).

61. Kresse, G. \& Furthmuller, J. Efficient iterative schemes for ab initio totalenergy calculations using a plane-wave basis set. Phys. Rev. B 54, 11169 (1996).

62. Heyd, J. \& Scuseria, G. E. Efficient hybrid density functional calculations in solids: Assessment of the Heyd-Scuseria-Ernzerhof screened Coulomb hybrid functional. J. Chem. Phys. 121, 1187 (2004)

63. Heyd, J., Peralta, J. E., Scuseria, G. E. \& Martin, R. L. Energy band gaps and lattice parameters evaluated with the Heyd-Scuseria-ernzerhof screened hybrid functional. J. Chem. Phys. 123, 174101 (2005).

64. Shao, Y. et al. Advances in methods and algorithms in a modern quantum chemistry program package. Phys. Chem. Chem. Phys. 2, 3172-3191 (2006)

\section{Acknowledgements}

This collaborative project was supported by the Department of the Navy, Office of Naval Research (Award No. N00014-14-1-0580). Synthesis of p-SIDT(FBTTh $)_{2}$ was supported by the Office of Naval Research (N-00014-04-0411). HR-TEM and FIB analysis were conducted in the MRL Shared Experimental Facilities at UCSB. The MRL Shared Experimental Facilities are supported by the MRSEC Program of the NSF under Award No. DMR 1121053; a member of the NSF-funded Materials Research Facilities Network (www.mrfn.org). S.R. and D.N. acknowledge financial support by the German Ministry of Science and Education (Project UNVEIL, FKZ 13N13719). The high resolution TDCF setup was funded by the Deutsche Forschungsgesellschaft (INST 336/94-1 FUGG)

\section{Author contributions}

N.A.R. fabricated the samples and devices used for all measurements. V.S. completed all $\mathrm{X}$-ray diffraction measurements and analysis for structural characterization. J.A.L. and C.J.T. performed the HR-TEM imaging and FIB processing for structural characterization. N.A.R. completed $J-V$, EQE, EL, IQE, EQE $\mathrm{EL}_{\mathrm{L}}$, UPS, AFM, absorption, temperaturedependent and bias-dependent EQE characterization, and the corresponding analyses. S.R. carried out $J-V, \mathrm{EQE}, \mathrm{EL}, \mathrm{EQE}_{\mathrm{EL}}$, temperature-dependent $V_{\mathrm{OC}}$, TDCF, and biasdependent EQE characterization, and the corresponding analyses. Y.T.S., H.L., and V.C. carried out the Monte Carlo and molecular dynamics simulations and the electronic structure calculations. X.L. synthesized the p-SIDT $\left(\mathrm{FBTTh}_{2}\right)_{2}$ used in these studies G.C.B., J.L.B., M.F.T., D.N., T.Q.N. supervised the project. N.A.R. prepared the manuscript, and all authors contributed to the writing and fine-tuning of the final manuscript.

\section{Additional information}

Supplementary Information accompanies this paper at doi:10.1038/s41467-017-00107-4.

Competing interests: The authors declare no competing financial interests.

Reprints and permission information is available online at http://npg.nature.com/ reprintsandpermissions/

Publisher's note: Springer Nature remains neutral with regard to jurisdictional claims in published maps and institutional affiliations.

Open Access This article is licensed under a Creative Commons Attribution 4.0 International License, which permits use, sharing, adaptation, distribution and reproduction in any medium or format, as long as you give appropriate credit to the original author(s) and the source, provide a link to the Creative Commons license, and indicate if changes were made. The images or other third party material in this article are included in the article's Creative Commons license, unless indicated otherwise in a credit line to the material. If material is not included in the article's Creative Commons license and your intended use is not permitted by statutory regulation or exceeds the permitted use, you will need to obtain permission directly from the copyright holder. To view a copy of this license, visit http://creativecommons.org/ licenses/by/4.0/.

(c) The Author(s) 2017 